\title{
Analysis of Automatically Extracted Hallux Valgus Angles Using Markerless 3D Foot Scans From North America, Europe and Asia
}

Yang Jiao ( $\nabla$ yang.jiao@volumental.com )

Volumental AB https://orcid.org/0000-0003-4771-0088

Sašo Džeroski

Jozef Stefan Institute

Ales Jurca

Volumental $A B$

\section{Research}

Keywords: Hallux Valgus Angle, 3D scans, footwear

Posted Date: November 1st, 2021

DOI: https://doi.org/10.21203/rs.3.rs-1008905/v1

License: (c) (i) This work is licensed under a Creative Commons Attribution 4.0 International License.

Read Full License 


\title{
Analysis of automatically extracted hallux valgus angles using markerless 3D foot scans from North America, Europe and Asia
}

\author{
Yang Jiao ${ }^{1 *}$, Sašo Džeroski ${ }^{3,2}$ and Ales Jurca ${ }^{1,2}$
}

${ }^{*}$ Correspondence:

yang.jiao@volumental.com

${ }^{1}$ Volumental AB, Stockholm,

Sweden

Full list of author information is

available at the end of the article

\begin{abstract}
Background: The forefoot is the foot most affected part by ill-fitting shoes. Footwear fitting standards have the measurements of length, width and arch length. Toe shape has not yet been used in footwear measurement. This study aims at investigating the variation in toe shape, as measured by the hallux valgus angle.
\end{abstract}

Methods: An automatic and reproducible hallux valgus angle measuring method using 3D foot scans with no palpation markers is proposed and applied to about half a million samples collected across North America, Europe and Asia. The measuring method is robust, it can detect the medial contour along the proximal phalanx even in extreme cases.

Results: The hallux valgus angle has a normal distribution with long tails on both sides in the general population. In the three regions (North America, Europe, Asia), the mean and standard deviation of this angle are $9^{\circ} \pm 6^{\circ}$, $8^{\circ} \pm 6^{\circ}, 12^{\circ} \pm 6^{\circ}$ for males and $11^{\circ} \pm 7^{\circ}, 12^{\circ} \pm 7^{\circ}, 16^{\circ} \pm 7^{\circ}$ for females.

Conclusions: The hallux valgus angle has a broad distribution in the general population. Females have larger hallux valgus angle than males, and people from Asia have larger hallux valgus angle than people from North America and Europe.

Keywords: Hallux Valgus Angle; 3D scans; footwear

\section{Introduction}

Human foot morphology is affected by wearing footwear. The hallux valgus angle (HVA), also called first metatarsophalangeal angle, first toe angle, or toe \#1 angle, is the most significantly different measurement between habitually shod and barefoot population [1]. Shoe toe box shape [2], opened-toed versus closed-toed shoes [3] and shoe fitting [4] are essential factors influencing HVA to increase. Hallux valgus angle is related to plantar pressure during walking and running [1]. Hallux valgus angle larger than $15^{\circ}$ is an indicator of hallux valgus (HV), the most common forefoot deformity, which will cause foot pain, and can alter foot function [5]. Hallux valgus has a multifactorial etiopathogenesis and is a progressive deformity. Is inadequate footwear one of the initial causes, or is it more of a factor that causes the progression of the deformity, which is still under debate $[6,5]$. Footwear design should consider flexibility and support. A toe box and a sole should allow normal metatarsal phalangeal joint movement [7], and offer forefoot support [1].

Radiography during weight-bearing is the gold standard to measure HVA [8]. It measures the angle formed between a longitudinal bisection of the first metatarsal 
and a longitudinal bisection of the base of the proximal phalanx. This procedure is invasive and not suggested for healthy people.

Measurement by goniometer is a commonly used non-invasive method $[9,10$, $11,12,13]$. The measurement is applied to a barefoot person with weight equally distributed on both feet. One arm of the goniometer is brought against the midline of the medial surface of the hallux. The hinge of the goniometer is located over the first metatarsophalangeal joint, while the other arm is brought against the midline of the medial surface of the first metatarsal [9]. The agreement of goniometer and radiographic measurement resulted in an intraclass correlation coefficient (ICC) of 0.81 (95\% confidence interval 0.76 to $0.86 ; p<0.001$ ) [11]. The measurement is operator-dependent and requires expert and special training.

With the development of 3D scanning and CAD tools, efforts have been made to utilize these technologies in foot morphology studies and footwear design. Previous studies that have quantitatively evaluated the HVA either need palpation landmarks of feet before scanning $[14,15,11,3]$, or manual adjustments of tangent lines on 3D scans $[12,16,17]$.

These processes require the measurer to be well trained and limit the reproducibility and scale of these studies. These studies consisted of no more than 1662 subjects.

Large scale surveys have utilised interviews or questionnaires to gather self-report data $[18,19,20]$. The largest sample was 197,422 participants in a US national health survey [19]. The quality of self-assessment is hard to control. The surveys focused on the prevalence and severity of hallux valgus deformity. No numerical angular data was collected.

Footwear design should also consider the variation from different geographies. Previous studies have analyzed HVA variation in different groups of subjects. Some of the studies focused on groups of subjects having extremely different shoe wearing habits, and revealed that the habitually shod population has significantly larger HVA than the barefoot population $[1,17]$. Females in the shod group have significantly larger HVA than males [21, 22]. Kusumoto et al. compared Filipino and Japanese females, Lee et al. compared Taiwanese and Japanese females. Both studies found significant differences in HVA between the two groups [23, 24]. To date, there have not been any documented large scale studies analyzing HVA values of subjects from multiple continents.

In this study, we propose and investigate a novel HVA measuring method. Our method uses 3D foot scans with no palpation landmarks and can be easily scaled up to a large number of participants. Using this method, we analyse the HVA variation of about half a million 3D foot scans.

\section{Method}

\section{Data collection}

This study was conducted in compliance with the Helsinki Declaration, and has been approved by the Swedish Ethics Review Authority (reference No. 2019-03243). The study was conducted on 3D foot scans collected by Volumental AB prior to the study. The subjects in the study are customers of 447 brick and mortar stores of footwear brands and retailers that are using a Volumental foot scanning solution. The scan acquisition process is described by Jurca et al. [25]. The demographic 
information collected for each customer included sex and age group (adult or child). All scans included in our study originated from North America, Europe and Asia, for adults taken during the time interval between October 2018 to December 2019 were within the length classes between $200 \mathrm{~mm}$ to $320 \mathrm{~mm}$ (with intervals of $5 \mathrm{~mm}$ as in Mondopoint shoe size system). Foot scans with instep height lower than $30 \mathrm{~mm}$ or higher than $90 \mathrm{~mm}$ were excluded from the analysis in order to remove scans of hands, feet with shoes, or with pant legs not rolled up. Foot scans that were outliers were visually investigated, and abnormal scans were excluded from the analysis. Excluded were, for example, scans of objects other than human feet, unfinished scans, scans of feet with missing toes, scans of feet standing on toes or the lateral side, or scans of left and right feet in touch with each other. Table 1 shows the number of scanned feet in each group. The unit of analysis in this study is a 3D foot scan. As the data was collected in a retail environment, the 3D scans were collected with or without socks. Approximately $7 \%$ of the scans are from bare feet, while the others are scans of feet with socks.

Table 1 Number of scanned feet per region and sex used in this study.

\begin{tabular}{lrrrr}
\hline & North America & Europe & Asia & Sum \\
\hline Male & 196,332 & 37,574 & 42,872 & 276,778 \\
Female & 152,654 & 24,574 & 29,522 & 206,750 \\
All & 348,986 & 62,148 & 72,394 & 483,528 \\
\hline
\end{tabular}

Hallux valgus angle measurement

In this study, we proposed a new method to extract HVA from 3D foot meshes. In addition, the foot dimensions of foot length, width, heel width and instep height were extracted. Foot orientation, foot outline extraction, and measurement extraction algorithms were the same as used by Jurca et al. [25]. Hallux valgus angle was measured as the angle between a base line parallel to the first metatarsal, and a hallux medial line parallel to the hallux proximal phalanx, similar to the arms in goniometer measurement [9].

The base line was defined by the calculated markers used in foot width and heel width measurements ( $P$ and $H$ as presented in Fig. 1). Point $\mathrm{P}$ is the extreme point of the medial part of the foot outline in the region between $65 \%$ and $80 \%$ of the foot length, i.e., the medial marker defining foot width [25]. Point $\mathrm{H}$ is the medial point on the foot outline cross section that was perpendicular to the main foot axis (y), located at $15 \%$ of the foot length, i.e., the medial marker defining heel width. The base line is the line connecting $P$ and $H$.

The hallux medial line is the line parallel to the longest line segment on the hallux convex curve. As shown in Fig. 1, $T$ is the extreme point of the anterior part of the foot outline. First, we generate a convex hull of the outline between points $P$ and $T$. We then approximate the convex curve by connected line segments with endpoints as a subset of the convex curve vertices. The error of each segment from the hallux convex curve is under a given error bound [26]. We adopt the optimal method described by Chan et. al. [27] to find all line segments that satisfy the error criteria on a convex curve, then pick the longest one to present the line parallel to the proximal phalanx. 
Figure 1 The measurement of hallux valgus angle (HVA) from a 3D foot scan outline. The gray curve is the hallux convex curve, with cross markers being the vertex on the convex curve.

The angle between this hallux medial line and the base line is then recorded as the HVA. This method was developed empirically based on a large number of observations. It is based on 3D shape only, without any anatomical palpation or manually post processing.

\section{Reliability of the measurement methods}

We investigated the outliers having HVA larger than $60^{\circ}$. They correspond to feet with a longer second toe, and the longest segment being the line connecting tips of the first and the second toe, representing less than $1 \%$ of all the feet. We repeated the process of HVA calculation for these outliers, after removing the curve between the first and the second toe ( $T T^{\prime}$ in Fig. 2) from the hallux convex curve.

The segment error criteria from the hallux convex curve was chosen as $0.2 \%$ foot length. In the majority of cases, the method using this parameter value accurately locates the medial contour of the proximal phalanx. While this parameter varied from 0 to $1 \%$ foot length, $93 \%$ of the feet had calculated HVA change less than $5^{\circ}$.

Fig. 3 is an example of constructing the hallux medial line with error tolerance from 0 to $1 \%$ foot length. Error tolerance of $1 \%$ foot length is too big, as there is a clear deviation from the line segment to the hallux convex curve.

\section{Variability of the hallux valgus angle}

To visualize the results of the measuring method, we randomly sampled left foot scans with HVA increasing in $5^{\circ}$ steps (Fig. 4). The base lines and the hallux medial lines are shown with blue and red lines. All of the plots are obtained by following the method described above, without any manual modification. The measured angles 


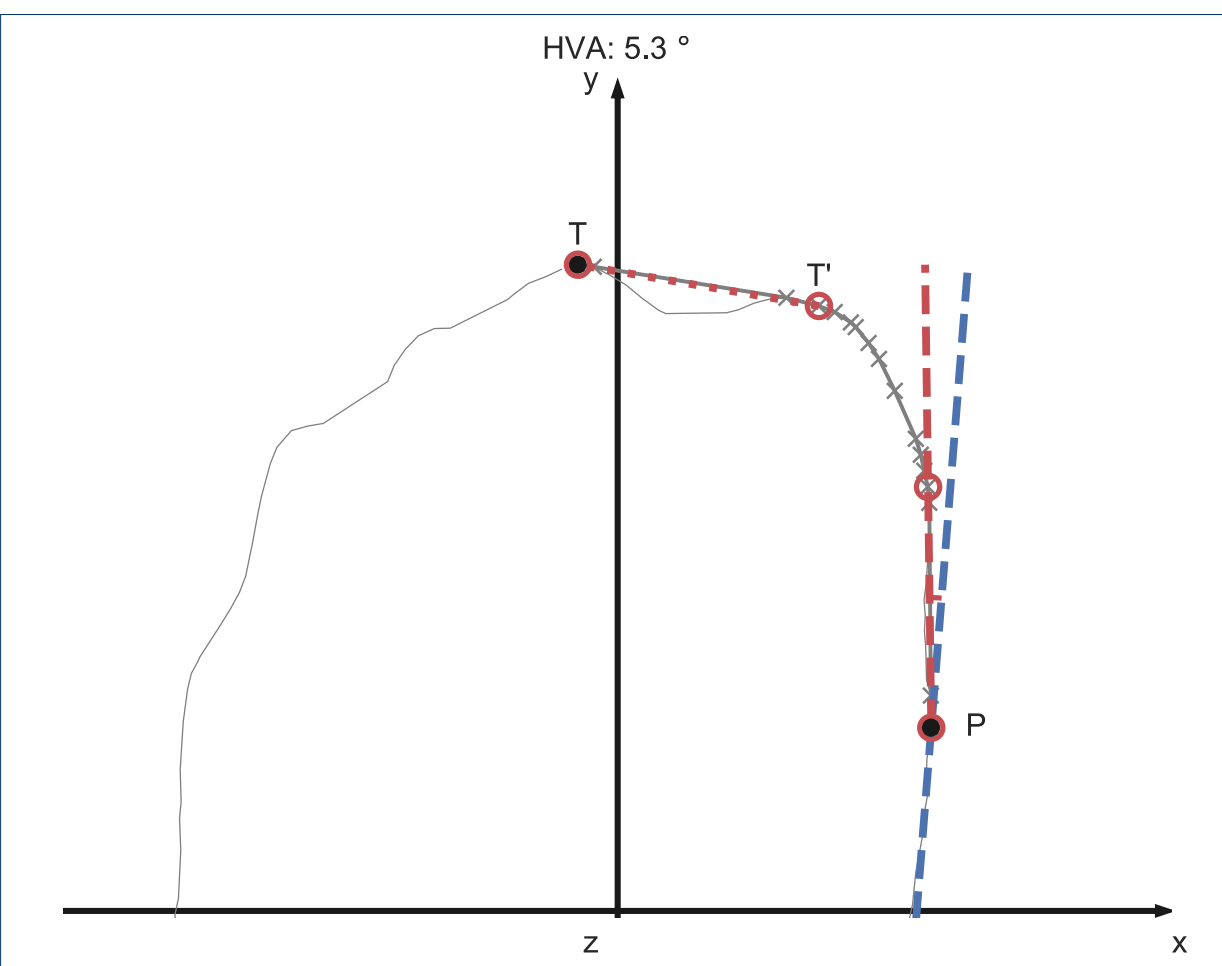

Figure 2 An example of the longest line segment between $P, T$ (dotted line) connecting the first and the second toes. Since its angle $\left(84.8^{\circ}\right)$ to the baseline is larger than the angular criteria of $60^{\circ}$, the longest line segment between $P, T^{\prime}$ is chosen to represent the line parallel to the first proximal phalanx. The blue dashed line is the baseline and the HVA (between the two dashed lines) is $5.3^{\circ}$.

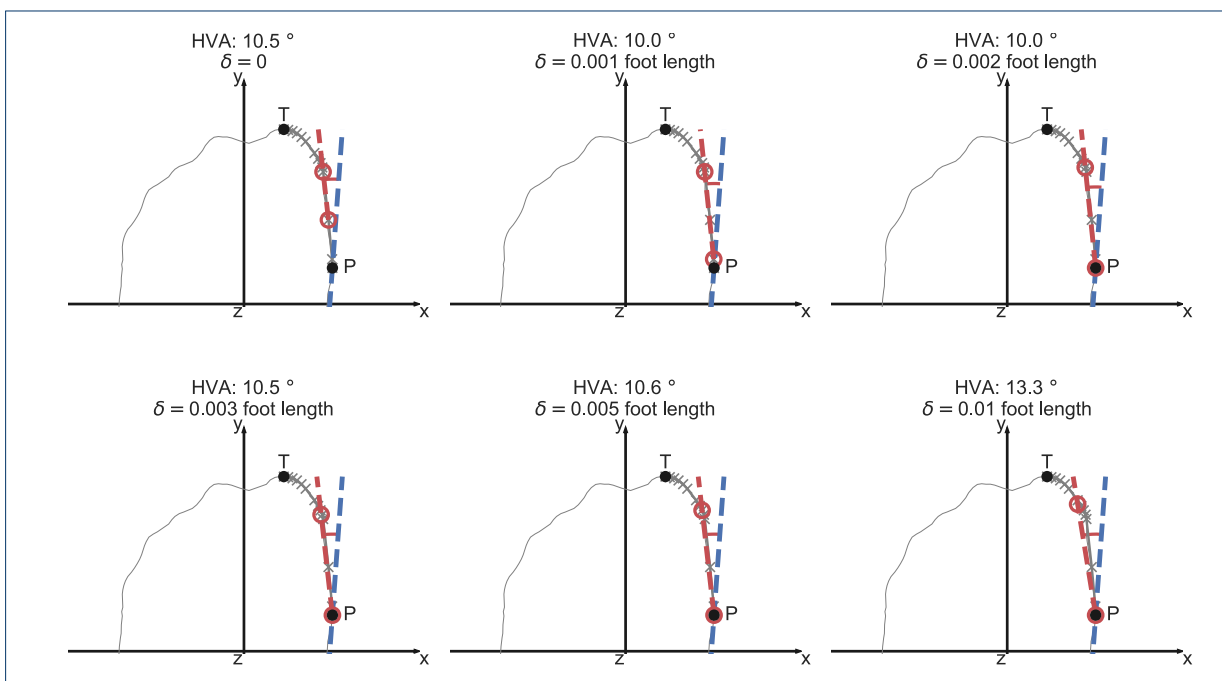

Figure 3 Hallux valgus angle as measured using the line segment error tolerance from 0 to $1 \%$ foot length. The red circles are the line segment endpoints. The red line connecting the circles is the hallux medial line. The blue line is the baseline.

present the trend in HVA, even in extreme cases as HVA of minus values or values in excess of $40^{\circ}$. 


\section{Result}

Dispersion of HVA in the general population

The HVA values in the general population follow a normal distribution with a significant skew and kurtosis. Fig. 5 shows the HVA of males and females in North America, Europe and Asia. The standard deviations for each of the three regions are approx. $6^{\circ}$ in males and $7^{\circ}$ in females (Table 2). The HVA values are positively skewed with skew parameters in ranges of $0.5-0.8$. They have extended tails on both sides, with positive kurtosis parameters in ranges of $1-3$. In the following analysis, foot scans are divided into groups by region and sex.

The discrepancies in HVA values between regions and groups were confirmed by a statistical significance test using Cohen's $d$ [28]. This test evaluates the effect size of the intergroup difference in means relative to the pooled standard deviation. Females had larger HVA values than males. The difference in mean HVA values between females and males was the smallest in North America, and the largest in Asia. The values of Cohen's $d$ and the effect size are given in Table 2 .

People from Asia have larger HVA than people from North America and Europe. The differences between North America and Europe were trivial for both genders. The differences of North America and Europe to Asia had medium effect size (Table $3)$.

Table 2 Differences among HVA values between shapes for the three regions, groups as measured by Cohen's $d$ [28].

\begin{tabular}{lrrr}
\hline & $\begin{array}{c}\text { North America } \\
(\text { mean } \pm \text { std })\end{array}$ & $\begin{array}{c}\text { Europe } \\
(\text { mean } \pm \text { std })\end{array}$ & \multicolumn{1}{c}{$\begin{array}{c}\text { Asia } \\
\text { (mean } \pm \text { std })\end{array}$} \\
\hline Male & $8.7 \pm 6.3$ & $8.0 \pm 6.4$ & $12.2 \pm 5.9$ \\
Female & $11.5 \pm 7.2$ & $11.6 \pm 7.0$ & $16.6 \pm 7.0$ \\
\hline Cohen's $d$ & 0.41 & 0.55 & 0.69 \\
Effect size & Small & Medium & Medium \\
\hline
\end{tabular}




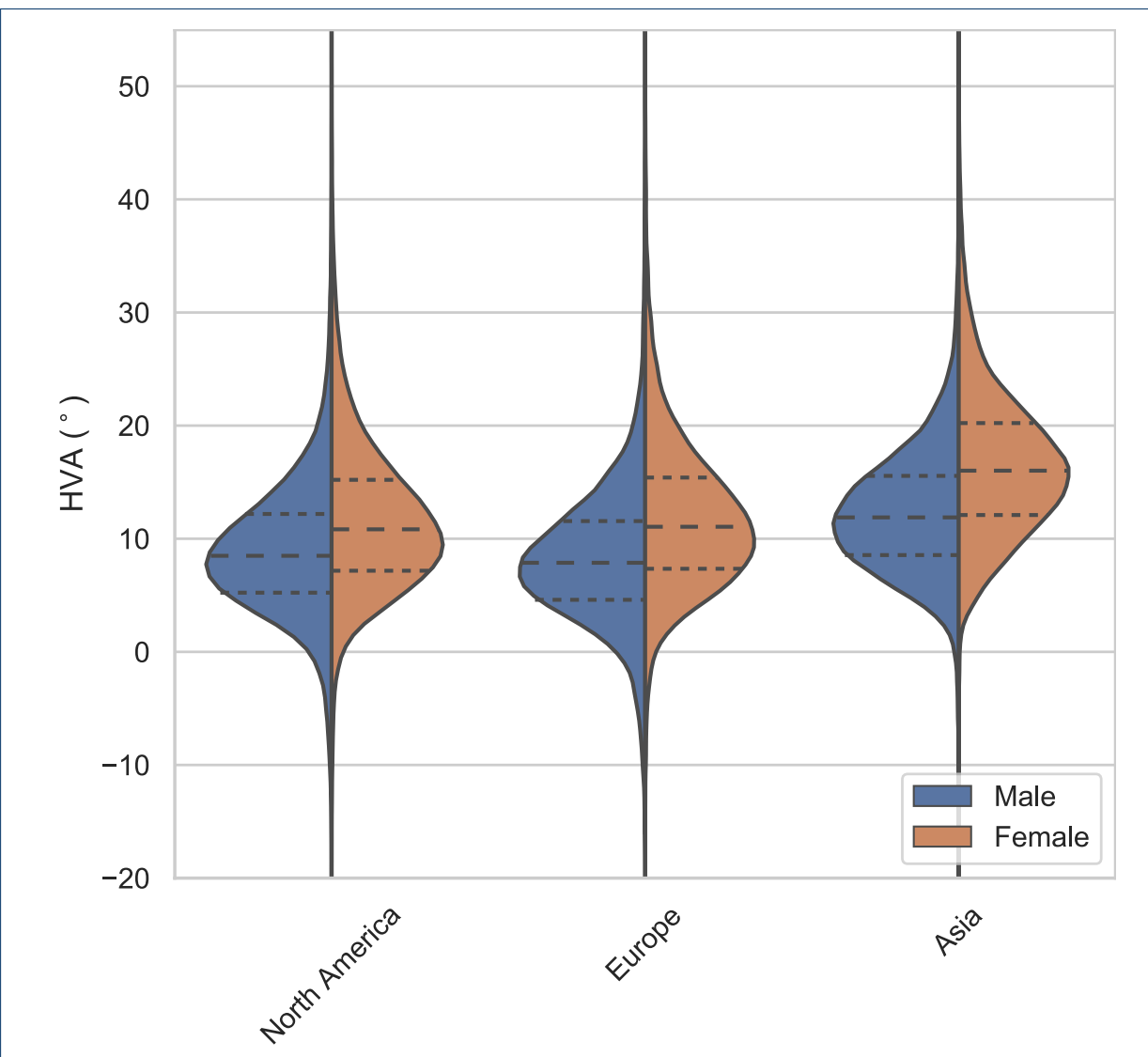

Region

Figure 5 The dependence of hallux valgus angle (HVA) on region and sex. Hallux valgus angle values for females are larger as compared to males. People from Asia have larger HVA values than people from North America and Europe.

Table 3 Pairwise differences of HVA values between regions/groups for both sexes as measured by Cohen's $d$.

\begin{tabular}{|c|c|c|c|c|}
\hline & & North America - Europe & North America - Asia & Europe - Asia \\
\hline \multirow{2}{*}{ Male } & Cohen's $d$ & 0.11 & 0.55 & 0.68 \\
\hline & Effect size & Trivial & Medium & Medium \\
\hline \multirow{2}{*}{ Female } & Cohen's $d$ & 0.02 & 0.71 & 0.69 \\
\hline & Effect size & Trivial & Medium & Medium \\
\hline
\end{tabular}

Hallux valgus angle did not exhibit strong correlation with foot length (Fig. 6). We divided the feet into foot length classes with a $5 \mathrm{~mm}$ increment (according to the mondopoint sizing system). Feet were classified to the length classes closest to their length. Feet classified to length classes $200 \mathrm{~mm}$ to $320 \mathrm{~mm}$ were included in this study. The foot length in each region and sex group has a normal probability distribution. The most frequently occurring length classes for male customers were $270 \mathrm{~mm}$ for scans in North America and Europe, and $255 \mathrm{~mm}$ for scans in Asia. For female customers [25], the most common length classes were $245 \mathrm{~mm}$ for scans in North America and Europe, and $235 \mathrm{~mm}$ in Asia. Only a small number of feet were classified into the extreme length classes at both ends, causing the wide confidence intervals for HVA values in these classes. The mean HVA values of females from Europe decrease with foot length when the latter is in the range $220 \mathrm{~mm}$ to $300 \mathrm{~mm}$. 


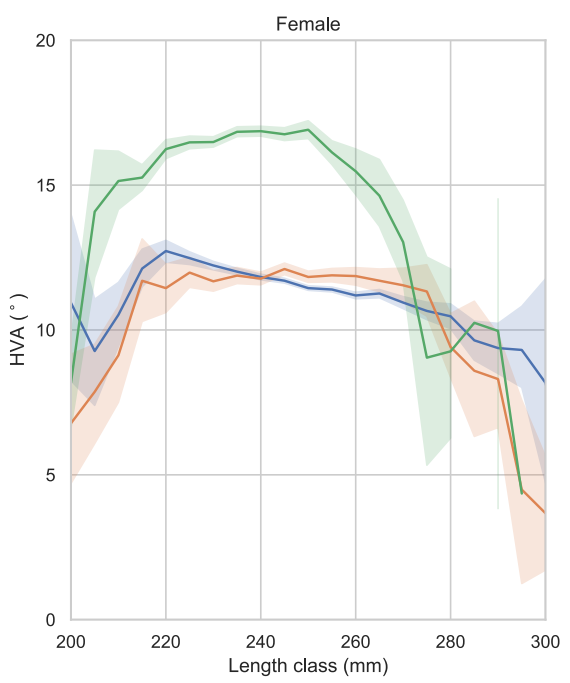

Figure 6 Mean hallux valgus angle (HVA) of each length class with the corresponding $95 \%$ confidence interval.

We further investigated the correlation of HVA with foot width (Fig. 7). The foot width standard deviations in the most frequently occurring length classes were $4.66 \mathrm{~mm}, 4.46 \mathrm{~mm}$ and $4.13 \mathrm{~mm}$ for male in North America, Europe and Asia. The corresponding values for females were $4.36 \mathrm{~mm}, 4.23 \mathrm{~mm}$ and $4.14 \mathrm{~mm}$. These deviations are close to the width step increment of $4.76 \mathrm{~mm}$ defined by the American standard shoe sizing system [29]. We divided feet in each length class into narrow, medium and wide foot width sub-classes. The medium sub-class was defined to have the same median as the entire length subgroup, and a $4.76 \mathrm{~mm}$ width range. The wide and narrow foot width sub-classes were defined to include feet with width above and under the medium sub-class, respectively. The medium width sub-class contains $40 \%$ of the feet.

The mean HVA values and standard deviations for each width group are presented in Table 4. The differences between mean HVA values of width groups are statistically significant for every geography and both genders. However, the dispersion within each width group is much larger than the differences between mean HVA values of width groups (Fig. 7) The effect size of difference in HVA among foot width sub-classes was trivial in males, and small in females (Table 5).

Table 4 The mean and standard deviations of HVA values in each foot width group for males and females in each of the three regions.

\begin{tabular}{llrrr}
\hline & & North America & Europe & Asia \\
\hline \multirow{3}{*}{ Male } & narrow & $7.9 \pm 5.5$ & $7.2 \pm 5.6$ & $11.6 \pm 5.5$ \\
& medium & $8.6 \pm 6.0$ & $8.0 \pm 6.0$ & $12.1 \pm 5.6$ \\
& wide & $9.6 \pm 7.4$ & $8.9 \pm 7.4$ & $12.9 \pm 6.7$ \\
\multirow{2}{*}{ Female } & narrow & $9.9 \pm 5.9$ & $10.0 \pm 5.9$ & $14.8 \pm 5.9$ \\
& medium & $11.2 \pm 6.5$ & $11.4 \pm 6.5$ & $16.2 \pm 6.4$ \\
& wide & $13.7 \pm 8.6$ & $13.8 \pm 8.6$ & $18.9 \pm 8.3$ \\
\hline
\end{tabular}

The association of HVA with instep height was evaluated using Pearson's correlation coefficients $(r)$. Instep height was first normalized by foot length. HVA was negatively, weakly correlated with normalized instep height $(r=-.15)$. 

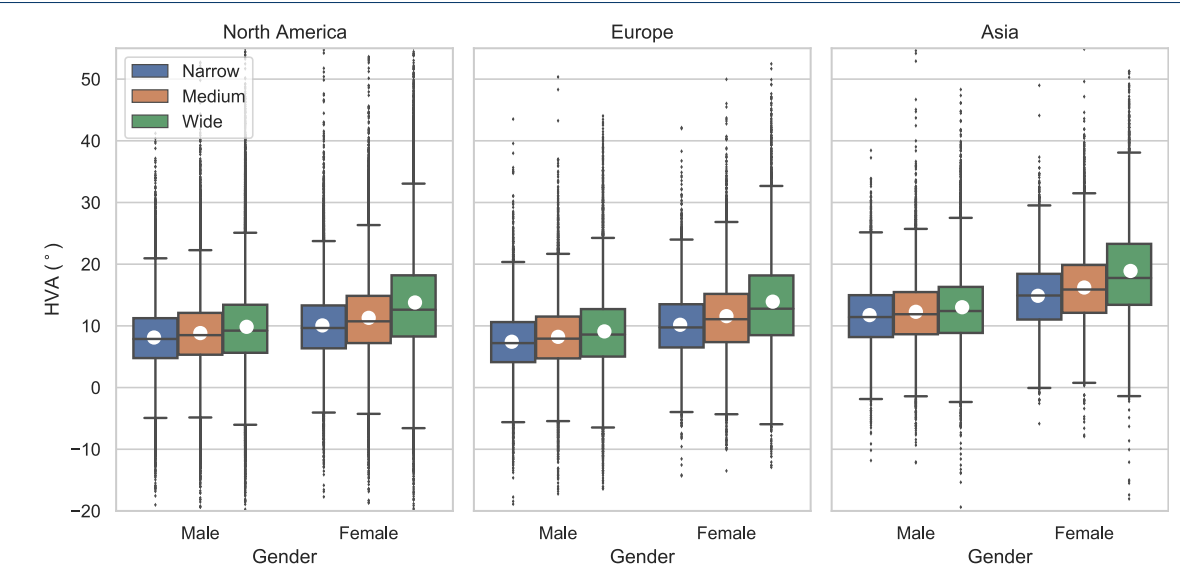

Figure 7 The distribution of hallux valgus angle (HVA) values in each width group for males and females in each of the three regions.

Table 5 Differences among narrow-medium and medium-wide width groups measured by Cohen's $d[28]$.

\begin{tabular}{llrrr}
\hline & & $\begin{array}{r}\text { North America } \\
\text { narrow-medium/ } \\
\text { medium-wide }\end{array}$ & $\begin{array}{r}\text { Europe } \\
\text { narrow-medium/ } \\
\text { medium-wide }\end{array}$ & $\begin{array}{r}\text { Asia } \\
\text { narrow-medium/ } \\
\text { medium-wide }\end{array}$ \\
\hline \multirow{2}{*}{ Male } & Cohen's $d$ & $0.12 / 0.15$ & $0.12 / 0.14$ & $0.10 / 0.12$ \\
& Effect size & Trivial/Trivial & Trivial/Trivial & Trivial/Trivial \\
\multirow{2}{*}{ Female } & Cohen's $d$ & $0.20 / 0.34$ & $0.23 / 0.32$ & $0.23 / 0.37$ \\
& Effect size & Small/Small & Small/Small & Small/Small \\
\hline
\end{tabular}

\section{Discussion}

The aim of this study was to develop a novel method of measuring HVA that could be used on large datasets of 3D foot scans, and to analyze HVA using a dataset of about half a million male and female 3D foot scans collected in a retail environment. Compared to existing methods of measuring HVA (radiographic or goniometer), this method is noninvasive and faster. It is completely automatic; it does not require any manual placing of markers on specific parts of the foot, and it does not require any manual positioning of markers or lines on a 3D foot scan; therefore, no specific training of measurers are required.

A major limitation of this work is the lack of a comparison to the existing methods. We were only able to qualitatively evaluate the accuracy of the measured HVA values by visually inspecting a large number of images as presented in Fig. 3 and 4. In order to quantitatively compare our method with the existing gold standard of HVA measurement, we would need to obtain a dataset of HVA measurements of a group of subjects based on x-ray images and 3D foot scans; however, collecting $\mathrm{x}$-ray images was beyond the scope of this research.

Another limitation of the proposed method was the step of fine-tuning the error tolerance parameter, which impacts the determination of the hallux medial line. This parameter was set to $0.2 \%$ of foot length by visually inspecting the results of the method using several values of the parameter. We chose the value of the parameter that calculated hallux medial lines similar to how an arm of a goniometer would be placed against the first toe. In order to evaluate the impact of the error tolerance parameter on the results, we calculated the mean HVA values for every group of subjects using each of the following parameter values: $0.1 \%, 0.2 \%, 0.3 \%$ 
and $0.5 \%$ of foot length. For female groups in length classes $220 \mathrm{~mm}$ to $280 \mathrm{~mm}$, and male groups in length classes $245 \mathrm{~mm}$ to $305 \mathrm{~mm}$, the mean HVA values varied less than $1.5^{\circ}$. This indicates that the chosen value of the error tolerance parameter does not have a substantial impact on the mean HVA values. Having a dataset of HVA measurements using both the gold standard method and our method would allow us to fine-tune this parameter so that we would achieve the highest possible agreement between the results of both methods.

Regardless of the above mentioned limitations of the proposed method, the possibility of using it on large datasets of 3D foot scans enables research of HVA at a much larger scale compared to existing methods. The number of subjects used for this study was orders of magnitude larger than any previous study of HVA measurements. This study had been conducted using male and female 3D foot scans from 3 geographical regions, which allowed comparisons of HVA values between these groups of customers.

The main finding of this study is a large dispersion of HVA values for both genders, and in all 3 geographical regions. It is not clear how different HVA values impact the perceived fit of footwear; however, it is very likely that a specific forefoot shape of a shoe does not fit to the wide range of HVA values observed in this study. Further studies are needed to increase the understanding of the optimal forefoot shape of a shoe for a specific HVA value.

Unfortunately the dataset used in this study does not contain the age of the subjects; therefore, we were unable to study a relationship between HVA and the age of subjects. However, previous studies have reported that the development of hallux valgus is related to ages $[6,18,30,31]$. Some retailers are collecting the age of shoe shoppers in their customer relationship management (CRM) systems. Joining the age data from CRM systems with 3D foot scan data would enable a large-scale analysis of the relationship between HVA and age in the future.

Substantial differences between HVA of male and female feet have been reported by previous studies $[21,22]$. This study confirms that female customers have significantly larger HVA values than male customers in all three geographical regions. The largest difference between male and female customers has been observed in Asia. Analysis of the toe \#1 angle of Japanese subjects in [22] indicates that there was no significant difference between boys and girls in the age groups from 3 to 6 years of age, and for the large majority of age groups of children above 6 years of age and adults, female subjects had significantly higher HVA values compared to male. Kinz et al. [32] concluded that children with larger HVA values are associated with wearing shoes of insufficient length. One possible explanation for larger HVA values in female customers observed in this and previous studies could be that girls and women are more likely to wear too small shoes. However, additional research is needed to confirm this theory.

The results of this study have demonstrated higher mean HVA values of Asian customers compared to North American and European customers in both genders. The reason for this is not clear, it could be related to cultural differences in the perception of how shoes should fit between the 3 regions.

Hallux valgus angle is an important quantitative measurement of toe shape, and an indicator of foot health. A substantial part of the Asian customers ( $57 \%$ of females 
and $28 \%$ of males) have HVA larger than $15^{\circ}$, which is an indication of hallux valgus (HV). The share of customers with HV is lower in North America (26\% of females and $13 \%$ of males) and Europe (27\% of females and $11 \%$ of males); however, the values are still high, especially for female customers. All these numbers indicate that customers should pay more attention to wearing the proper size of footwear. Footwear producers should pay attention to designing anatomically shaped footwear to reduce the impact of poorly fitting shoes on feet.

Wider feet have larger mean HVA values in all regions and in both genders; however, the differences between the width sub-groups are trivial or small in all groups.

\title{
Conclusion
}

In this study, we proposed a new method of measuring HVA, and applied the method to a large dataset of 3D foot scans. The results of the study revealed the large dispersion of HVA across all 3 geographical regions and both genders. Customers in Asia have higher HVA values than customers in North America and Europe. Females have higher HVA values than males in all 3 regions. The proposed method for measuring HVA opens a new opportunity in studying the causal relation of HVA and shoe wearing habits on a large scale. Using this method with 3D scanning devices in footwear retail could increase the awareness of customers about their toe shapes, and increase the number of properly fitted shoes.

\author{
Acknowledgements \\ Not applicable. \\ Funding \\ Not applicable. \\ Abbreviations \\ Not applicable. \\ Availability of data and materials \\ The raw foot scan data that support the findings of this study are available from [third party name] but restrictions \\ apply to the availability of these data, which were used under license for the current study, and so are not publicly \\ available. The processed data are however available from the authors upon reasonable request and with permission \\ of [third party name]. \\ Ethics approval and consent to participate \\ This study was conducted in compliance with the Helsinki Declaration, and has been approved by the Swedish \\ Ethics Review Authority (reference No. 2019-03243). \\ Competing interests \\ The authors acknowledge financial interest in Volumental AB, which offers a 3D foot scanner as one of its products. \\ However, the results of this study are independent of the particular 3D scanning technology and device used to \\ collect the raw foot scan data. \\ Consent for publication \\ Participants' consent to publish was granted. \\ Authors' contributions \\ YJ developed the method, performed the data analysis, interpreted the results and prepared the manuscript. SD \\ helped with the interpretation of the results and the preparation of the manuscript. AJ designed the study and \\ helped with the interpretation of the results and the preparation of the manuscript. \\ Author details \\ ${ }^{1}$ Volumental AB, Stockholm, Sweden. ${ }^{2}$ Jozef Stefan International Postgraduate School, Ljubljana, Slovenia. ${ }^{3}$ \\ Jozef Stefan Institute, Ljubljana, Slovenia.
}




\section{References}

1. Mei, Q., Gu, Y., Xiang, L., Yu, P., Gao, Z., Shim, V., Fernandez, J.: Foot shape and plantar pressure relationships in shod and barefoot populations. Biomech. Model. Mechanobiol. 19(4), 1211-1224 (2020). doi:10.1007/s10237-019-01255-w

2. Branthwaite, H., Chockalingam, N., Greenhalgh, A.: The effect of shoe toe box shape and volume on forefoot interdigital and plantar pressures in healthy females. J. Foot Ankle Res. 6(1), 28 (2013) doi:10.1186/1757-1146-6-28

3. Martin-Casado, L., Barquín, C., Aldana-Caballero, A., Marcos-Tejedor, F., Aguado, X.: Environmental factors as a cause of differences in the feet of Ecuadorian children and its relation to their footwear. Children 8(6) (2021). doi:10.3390/children8060459

4. McRitchie, M., Branthwaite, H., Chockalingam, N.: Footwear choices for painful feet - An observational study exploring footwear and foot problems in women. J. Foot Ankle Res. 11(1), 23 (2018). doi:10.1186/s13047-018-0265-2

5. Ferreyra, M., Núñez-Samper, M., Viladot, R., Ruiz, J., Isidro, A., Ibañez, L.: What do we know about hallux valgus pathogenesis? : Review of the different theories. J. Foot Ankle 14(3), 223-230 (2020)

6. Menz, H.B., Marshall, M., Thomas, M.J., Rathod-Mistry, T., Peat, G.M., Roddy, E.: Incidence and progression of hallux valgus: A prospective cohort study. Arthritis Care Res. n/a(n/a) (2021). doi:10.1002/acr.24754

7. Davies, N., Branthwaite, H., Chockalingam, N.: Where should a school shoe provide flexibility and support for the asymptomatic 6- to 10-year-olds and on what information is this based? A Delphi yielded consensus. Prosthet. Orthot. Int. 39(3), 213-218 (2015). doi:10.1177/0309364614522684

8. Menz, H.B., Munteanu, S.E.: Radiographic validation of the manchester scale for the classification of hallux valgus deformity. Rheumatology (Oxford) 44(8), 1061-1066 (2005). doi:10.1093/rheumatology/keh687

9. Kilmartin, T.E., Bishop, A.: Hallux abductus measurement: Repeatability trials of a clinical measuring instrument. Chiropodist, 185-187 (1988)

10. Kilmartin, T.E.: The orthotic treatment of juvenile hallux valgus. PhD thesis, University of Nottingham (1994)

11. Janssen, D.M., Sanders, A.P., Guldemond, N.A., Hermus, J., Walenkamp, G.H., van Rhijn, L.W.: A comparison of hallux valgus angles assessed with computerised plantar pressure measurements, clinical examination and radiography in patients with diabetes. J. Foot Ankle Res. 7(1), 33 (2014). doi:10.1186/1757-1146-7-33

12. Zhou, J., Hlavacek, P., Xu, B., Chen, W.: Approach for measuring the angle of hallux valgus. Indian J. Orthop. 47(3), 278-282 (2013). doi:10.4103/0019-5413.109875

13. Choung, S.-D., Kang, S.-Y., Kim, M.-H., Weon, J.-H.: Reliability and validity of the goniometer for hallux valgus angle measurement. Phys. Ther. Korea 20(2), 46-51 (2013). doi:10.12674/ptk.2013.20.2.046.

14. Mits, S.D., Coorevits, P., Clercq, D.D., Elewaut, D., Woodburn, J.J., Roosen, P.: Reliability and validity of the Infoot 3D foot digitizer for normal healthy adults. Footwear Sci. 2(2), 65-75 (2010). doi: $10.1080 / 19424281003685694$

15. Mickle, K.J., Munro, B.J., Lord, S.R., Menz, H.B., Steele, J.R.: Foot shape of older people: Implications for shoe design. Footwear Sci. 2(3), 131-139 (2010). doi:10.1080/19424280.2010.487053

16. Saghazadeh, M., Kitano, N., Okura, T.: Gender differences of foot characteristics in older Japanese adults using a 3D foot scanner. J. Foot Ankle Res. 8(1), 29 (2015). doi:10.1186/s13047-015-0087-4

17. Shu, Y., Mei, Q., Fernandez, J., Li, Z., Feng, N., Gu, Y.: Foot morphological difference between habitually shod and unshod runners. PLoS One 10(7), 0131385 (2015). doi:10.1371/journal.pone.0131385

18. Nix, S., Smith, M., Vicenzino, B.: Prevalence of hallux valgus in the general population: A systematic review and meta-analysis. J. Foot Ankle Res. 3(1), 21 (2010). doi:10.1186/1757-1146-3-21

19. Wilder, M.H.: Prevalence of chronic skin and musculoskeletal conditions, united states, 1969. national center for health statistics. Vital Health Stat. 10(92), 1-65 (1980)

20. Adams, P.F., Benson, V.: Current estimates from the national health interview survey, 1990. Vital Health Stat. 10 181, 1-212 (1991)

21. Barnicot, N.A., Hardy, R.H.: The position of the hallux in West Africans. J. Anat. 89(3), 355-361 (1955)

22. Kouchi, M.: Foot dimensions and foot shape: Differences due to growth, generation and ethnic origin. Anthropol. Sci. 106(Supplement), 161-188 (1998)

23. Kusumoto, A., Suzuki, T., Kumakura, C., Ashizawa, K.: A comparative study of foot morphology between Filipino and Japanese women, with reference to the significance of a deformity like hallux valgus as a normal variation. Annals of Human Biology 23(5), 373-385 (1996). doi:10.1080/03014469600004622

24. Lee, Y.-C., Kouchi, M., Mochimaru, M., Wang, M.-J.: Comparing 3D foot shape models between Taiwanese and Japanese females. J. Hum. Ergol. (Tokyo) 44(1), 11-20 (2015)

25. Jurca, A., Žabkar, J., Džeroski, S.: Analysis of 1.2 million foot scans from North America, Europe and Asia. Sci. Rep. 9(1), 19155 (2019). doi:10.1038/s41598-019-55432-z

26. Imai, H., Iri, M.: Computational-geometric methods for polygonal approximations of a curve. Comput. Vis. Graph. Image Process. 36(1), 31-41 (1986). doi:10.1016/S0734-189X(86)80027-5

27. Chan, W.S., Chin, F.: Approximation of polygonal curves with minimum number of line segments or minimum error. Int. J. Comput. Geom. Appl. 06(01), 59-77 (1996). doi:10.1142/S0218195996000058

28. Cohen, J.: Statistical Power Analysis for the Behavioral Sciences, 2nd edn. (1988)

29. Ales, J., Zabkar, J., Dzeroski, S.: Foot width dispersion of male customers in the US and Canada. Footwear Sci. 11(sup1), 163-165 (2019). doi:10.1080/19424280.2019.1606307

30. Scott, G., Menz, H.B., Newcombe, L.: Age-related differences in foot structure and function. Gait Posture 26(1), 68-75 (2007). doi:10.1016/j.gaitpost.2006.07.009

31. Roddy, E., Zhang, W., Doherty, M.: Prevalence and associations of hallux valgus in a primary care population. Arthritis Care Res. 59(6), 857-862 (2008). doi:10.1002/art.23709

32. Kinz, W., Groll-Knapp, E., Kundi, M.: Hallux valgus in pre-school-aged children: The effects of too-short shoes on the hallux angle and the effects of going barefoot on podiatric health. Footwear Sci. 13(1), 29-42 (2021). doi:10.1080/19424280.2020.1853826 